\title{
Polymeric Piezoelectric Sensors and Remote Communication for Detection of Bruxism
}

\author{
Alexander Martinez, Antonio Barientos, Andrés Diaz, Pilar Lafont, \\ Inlián Colorado, Pedro Lnis Castedo, Roberto González \\ Universidad Politécnica de Madrid - Spain \\ alex.martinez@upm.es / adiaz@etsii.upm.es
}

\begin{abstract}
Active materials are capable of responding in a controlled way to different external physical or chemical stimulus by changing some of their properties These materials can be used to design and develop sensor's, actuators and multifunctional systems with a large number of applications for developing medical devices (for both surgery and implants).

On the other hand, Bruxism is a health problem consisting on grinding or tightly clenching the upper and lower teeth, and both phenomena lead to wear of the teeth and produce a noise during the night that is sufficiently loud to disturb the sleep of anyone sharing the bedroom. The tension produced also causes problems in the muscles tissues and other structures surrounding the jaw, ear pain, headaches, lesions to the teeth and disorders in the jaw joints. For an early, rapid, effective and economical diagnosis of bruxism, we propose the use of instrumented splints based on the use of active materials that detect and record the intensity and duration of interdentally pressure episodes.

This paper introduces the design, manufacture and testing of using instrumented splint for bruxism detection. The contribution of this work is basically focused on using piezoelectric polymeric sensors, taking advantage of their reduced thickness, and avoiding the alteration of the patient's bite. In addition, the system allows a quantitative assessment of intraoral pressure that consequently allows the diagnosis of bruxism behaviour at an early stage, aiming to perform corrective actions before irreversible dental wear appears. The first trials performed allowed to demonstrate the feasibility of the system, which also includes wireless communication capabilities for improving on telemedicine tasks.
\end{abstract}

\section{INTRODUCTION}

Intraoral pressnre sensing and measurement have several applications for dental, maxillofacial surgery, odontology, and especially for the diagnosis and monitoring of dental and occlusal pathologies. In addition, it is also nsed for the evaluation of post-surgical evolution and benchnnarking alternative treatments.

Due to the information derived from such measurementscan be efficiently used in clinical activities, the development of precise and repetitive sensing systems is especially valuable.

In the past two decades, several devices (and related patents) have been developed for the aforementioned tasks, allowing intraoral (almost always interdental) pressure sensing using different mechanical and electronic sensing principles.

From specialized literature revision, the next subsection presents the most relevant work in this field, and the contribntions of this work.

\section{A. Literature Review}

The use of extensiometric gauges has been reported and validated using in-vivo animal models, such as primates [1], porcine animals [2] and dogs [3]. These studies preceded the first in vivo trials in humans or related to veterinary surveys. In other cases, several devices have been nsed to assess the degree of patient satisfaction with dental prostheses, both fixed and removable, in order to validate therapeutic solutions and to propose improvements mentioned on [4]. [5], [6]

Among the most important surveys in relation to intraoral pressure sensing in humans, the bruxism and temporomandibular joint pathologies are the most current. Bruxism is a health problem that involves grinding or tightly clenching the upper and lower teeth. The resulting tension causes problems in the muscles, tissues and other structures surrounding the jaw; ear pain; headaches; lesions to the teeth and disorders of the jaw joints. All of these symptoms are collectively described as temporo-mandibnlar joint (TMJ) disorders or craniomandibnlar dysfnnction pain syndrome.

The phenomenon was introduced in the dental literature as "bruxomania" in 1907, by Pietkiewicz, who described the habit of teeth grinding. The term "bruxism" was introduced by Frohman in 1931, and in 1936, Miller proposed the use of the tem "bruxomania" for daytime grinding and "bruxism" for night-time grinding. The terms "Iraumatic neuralgia", "Karolyi effect" and "occlusive habit neurosis" have all been used to refer to some form of teeth grinding or clenching

According to stndies achieved by the Canadian Sleep Society [7], [8], [9], noctninal bruxism affects $8 \%$ of adults and $14 \%$ of children. A decrease in the population affected is apparently related to the age, in which about $3 \%$ of individuals over 60 have been affected. However, for other researchers [10]. [11], the prevalence is about $25 \%$.

In terms of gender differences in the affected popnlation, there is no general agreement since the literatnre reports contradictory data.

Measurements of intraoral pressure become especially relevant when are related to the stndy of bruxism, not jnst for evaluating the degree of such pathology but also for monitoring the evolution of patients and comparing different possible treatments. 
Special attention should be paid to results from some studies [12], [13], [14] that have tried to offer a quantitative definition of bruxism. Results from Nishigawa's study on the bite force produced during bruxism episodes suggested values as high as $1100 \mathrm{~N}$, exceeding the maximum voluntary bite force. Pressures on teeth surfaces can reach $40 \mathrm{MPa}$, sufficient to cause alarming levels of wear and even tooth breakage. In terms of the duration of bruxism episodes, an average about 7 seconds has been reported.

It is important to point out that everyone subconsciously clenches his or her teeth at some time during the day, and even this may be considered bruxism activity. However, the term bruxism is only correctly used when the duration and intensity of this activity have a bearing on dental wear and the development of TMJ problems.

\section{B. Contribution}

As previously mentioned, one of the main problems associated with the traditional diagnosis of bruxism is frequently made when the teeth are already highly worn and the prognosis of the illness is more severe. In order to make an earlier, more rapid, more effective and more economical diagnosis of bruxism, our research team proposes the use of instrumented splints for detecting and recording the intensity and duration of interdental pressure episodes.

We explain the design, manufacture and trials of our instrumented splint for diagnosing bruxism activity, according to Spanish patent initial objectives [15]. The splint offers additional telemedicine capabilities, such as remote patient monitoring and control. The miniaturised sensing device allows for a comfortable intra-mouth patient experience, does not cause alteration of the bite and improves the quality of final measurements.

The use of a wireless external interrogator/reader also allows the effective collection, treatment and study of the desired information in a more convenient way than with previous prototypes [16], [17] and the devices used in previously reported studies (both in animal models and in humans).

The splint was developed by researchers from the Universidad Politécnica de Madrid within the "FEMAB Project: Micro-instrumented Anti-bruxism Splint" subsidised by the Spanish Ministry of Education and Science as part of project PROFIT (Promotion of Technical Research), CIT020400-2005-17. Likewise, this work has been carried out in collaboration between the Universidad Politécnica de Madrid and Ibex Estética Dental S.L.

\section{ELECTROACTIVE POLYMERS AS SENSORS}

Traditionally discharge splints or protection devices are used to treat bruxism and prevent the associated dental wear. As a diagnostic device we propose introducing pressure sensors into a splint so that patient's bite episodes can be recorded and the extent of their pathology be assessed.

Polyvinylidene fluoride or PVDF $-\left(\mathrm{CH}_{2}-\mathrm{CF}_{2}\right)_{-\mathrm{n}}$ and its copolymers such as poly(vinilydenefluoride-trifluoroethylene) or $\mathrm{P}(\mathrm{VDF}-\mathrm{TrFE})$, are the Piezoelectric polymers with the largest number of industrial applications.

They posses partial crystalinity with an inactive amorphous phase and an elastic modulus between 1 and $10 \mathrm{GPa}$. Their use as actuators is limited by the need to apply high electric fields (around $20 \mathrm{~V} / \mu \mathrm{m}$ for a $3 \%$ deformation), but their use as pressure sensors is taking the place of traditionally used piezoelectric ceramic materials.

The use of this type of sensor was considered because of its reduced thickness, which does not cause any alteration to the patient's bite and because of its greater resistance and sensitivity compared to ceramic piezoelectrics. To make the sensors, we took PVDF $40 \mu \mathrm{m}$ thick sheets from Piezotech S.A. with Au-Pt coated electrodes. These sheets were cut, joined to the connecting wires and suitably encapsulated to protect them and then inserted into the splint. The sensors obtained are shown in Fig. 1.

Fig. 2-a shows the piezoelectric sensor layout. The charge displacement produced when a force is applied to the PVDF piezoelectric sensor can be represented using the equivalent electric circuit depicted in Fig. 2-b.

The validation of such behavior model, by comparing simulation results and "in vitro" trials, has previously been reported [16], [17].

Fig. 3 shows the first real trials carried out with the piezoelectric sensor connected directly to the oscilloscope when it was subjected to levels of pressure, with the help of a pneumatic workbench. However, signal conditioning is required for better "in vivo" results. This issue is explained in the next chapter in detail.

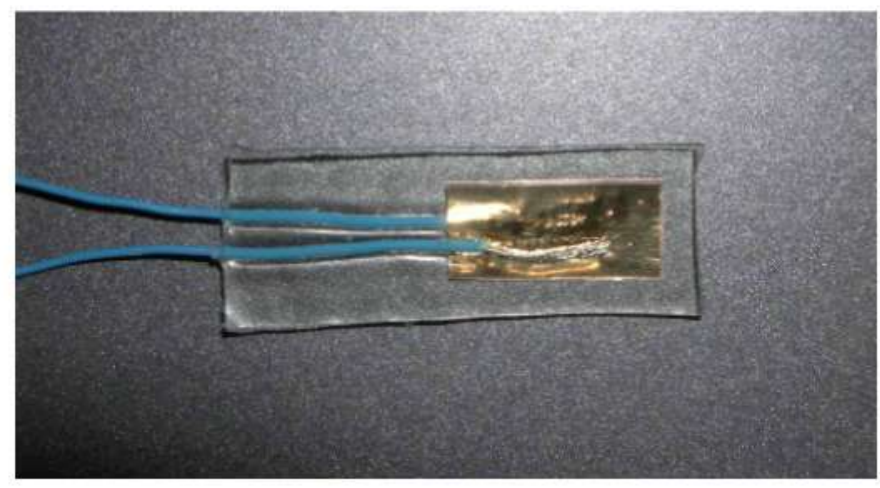

Fig 1. Piezoelectric sensors manufactured at the Product Development Laboratory of Universidad Politécnica de Madrid. 


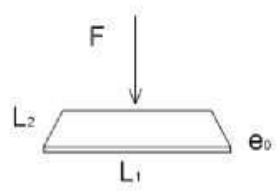

a)

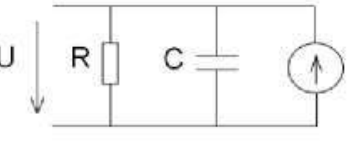

b)

Fig. 2. a) Piezoelectric Sensor. b) Electrical behavior circuit diagram of the piezoelectric sensor.

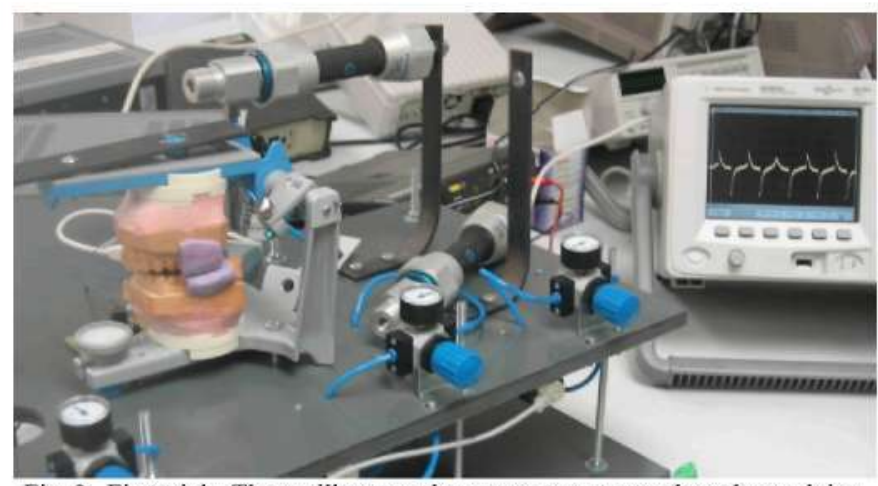

Fig. 3. First trials: The oscilloscope shows sensor output voltage by applying levels of pressure as input.

\section{SIGNAL CONDITIONING}

\section{A. Charge amplifier}

In one of the most typical working modes for using piezoelectric sensors, voltage between metallized sides of the sensor is forced to be $0 \mathrm{~V}$ and the generated charge is transferred to a known value condenser, in which tension is measured $(\mathrm{V}=\mathrm{Q} / \mathrm{C})$.

Such configuration corresponds to a charge amplifier, whose conditioning circuit is developed by using operational amplifiers that provides a low impedance output tension and can be subsequently processed with ease (due to being more stable against disturbances and noise).

The direct measurement of tension between metallized sides of the sensor raises some problems related to the fact that PVDF sensor's capacitance cannot easily be controlled, as a cause of its dependency on sensor's encapsulation and the spatial configuration of surrounding elements and connections, as well as the physical properties of the sensor film itself. Those variables would lead the system with high dispersion.

Therefore a charge amplifier was selected in order to avoid the explained problems, providing positive results using the both first prototypes and the final device. Nominal sensitivity of the combination "sensor - charge amplifier" has been set to 1.1 $\mathrm{mV} / \mathrm{N}$ and the nominal measurement range is from 0 to $850 \mathrm{~N}$. The conditioning circuit, with dimensions of $10.3 \mathrm{~mm} \times 8.9$ $\mathrm{mm} \times 1.0 \mathrm{~mm}$, is mounted between the sensor and the control and communication board.

Due to size limitations within the mouth, miniaturization was promoted and commercial integrates were used with a printed circuit of just $0.2 \mathrm{~mm}$ width. Additional miniaturization can be achieved for obtaining a system with final size similar to a tooth, but it would require extra funding and manufacturing technologies not advisable for prototype series.

Another design goal was to dramatically reduce battery consumption. On "sleep mode" board consumes around $4 \mu \mathrm{A}$. Such board is powered by a tension of $1.8 \mathrm{~V}$ generated by a LDO regulator incorporated to the control and communication board explained below.

\section{B. Control and communication board}

This board, with dimensions of $28.2 \mathrm{~mm} \times 18.0 \mathrm{~mm} \times 4$ $\mathrm{mm}$, contains all the intraoral splint electronics except for the charge amplifier. It includes the following subsystems::

- Anti-aliasing filter for both prototypes, and the final design, a $2^{\text {nd }}$ order low-pass filter, with a phase error of $0.05^{\circ}$ and a cut-off frequency of $9 \mathrm{~Hz}$, has been developed using a Sallen-Key cell.

- Control, communication, pre-processing and digitalization module based on the use of a Bluetooth WT32 from BlueGiga, developed from the BlueCore 5-nucleus from CSR. This module allows signal digitalization and preprocessing, communication's control and battery handle, which has promoted a high level of system integration.

- Supply of the digital I/O and the charge amplifier: An LDO precise regulator has been chosen for promoting low consumption. Both inner and outer regulators of the module allow to use the whole range of Li-Ion batteries, from $4.2 \mathrm{~V}$ to $3.1 \mathrm{~V}$, optimizing autonomy.

\section{- Energy source}

Due to the high consumption demand, the use of nonrechargeable batteries is not advisable. Although they usually have higher capacity than rechargeable ones, the periodical substitution would mean additional mechanical requirements for the splint, which would reduce product life.

Finally a Li-Ion rechargeable battery reference LIR2016 $(020 \mathrm{~mm} \times 1.6 \mathrm{~mm})$ with a capacity of $15 \mathrm{mAh}$ has been selected. Fig. 4 shows all components and final prototype of instrumented splint.

\section{COMMUNICATION WITH A REMOTE DEVICE}

The system objective is to capture sensor response data, process and send them to a remote device for treatment and analy sis of the information related to bruxist behaviour.

Communication between intramouth and external devices is based on the Bluetooth standard, which allows interchange of information between the instrumented splint and the patient's mobile phone with a target distance of around 30 meters.

It consists of a bidirectional communication between both devices, where both exchange information without interference of external devices. In addition, due to the point to point connection the system remains free of external wireless interference. 


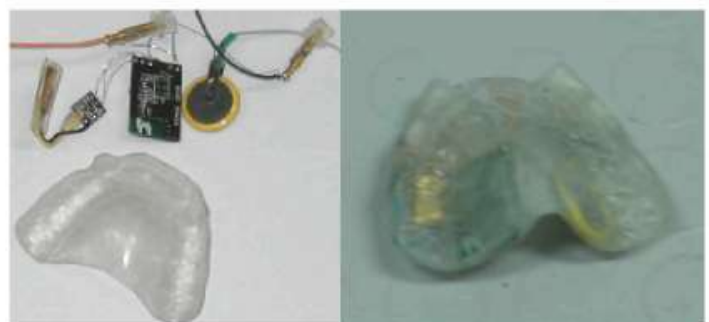

Fig.4. Instrumented splint before and after of assembly process

The system can be analyzed considering its three main capabilities:

- Data capture from the sensing system that detects and conditions the signal generated due to the bruxist episode.

- Wireless communication between the intramouth Bluetooth communication module and the external device (patient's mobile phone).

- Data storage and treatment within the mobile phone for subsequent medical/clinical study.

In this way we can study the system by dividing it into two main components:

- Microelectronic system for data gathering from the sensing system through an A/D converter. The device includes communication capabilities via Bluetooth.

- Data storage and treatment with help of an "ad-hoc" control software installed in the mobile phone.

\section{HARDWARE VALIDATION}

Several tests were conducted "in vivo" to verify the signal conditioning system functionalities. With these tests we collected sufficient and representative data of the bite forces of twelve volunteers (seven men and five women) aged between 20 and 30 years old.

All the tests consisted of four different types of experiments. Indications given to each volunteer before each test is summarized below, taking into account all the cases in which the state changed between tightening and loosening was determined by visual cues on a computer screen, given by the management software designed for that purpose.

- Measurement of maximal bite force $\left(\mathrm{F}_{\max }\right)$ : It consisted of three measurements of jaw clenching as hard as possible.

- Measurement of "grinding" episodes: We requested jaw clenching to volunteers, moving it sideways, bottom teeth rubbing against the upper. Nine measurements were performed with different force magnitudes, i.e. three of them with about $50 \%$ of their maximum force (measured in the previous test), another three about $25 \%$ and three more about $12 \%$ of that maximum force.

- Measurement of "clenching" episodes: Each volunteer pressed his jaw, applying pressure by joining the upper teeth against the bottom. Nine measurements were performed with different force magnitudes, three about $50 \%$, three about $25 \%$ and three about $12 \%$ of the maximum force previously measured.
- Measurement of "tapping" episodes: In this case, volunteers were suggested to "beat" their teeth with the maximum possible frequency for each person. Three measurements were performed with different force magnitudes: about $50 \%$, another about $25 \%$ and the last one about $12 \%$ of the maximum force previously measured.

Fig. 5 depicts an example obtained from experiments. On the left side the signals are plotted in absolute terms, and on the right side, the same signals are plotted in relative terms respect to its maximum force. The data also shows that the magnitude of the measured signals corresponds to the respective forces applied in each experiment, thereby validating the reliable operation of signal conditioning system.

\section{BRUXISM EPISODE DETECTION}

Once the proper functioning of the signal acquisition and processing system was verified, such signals were analyzed in order to develop an algorithm to detect events that may be consider "bruxists" events. This algorithm is based on the following approaches according to Fig. 6:

- If the magnitude of bite force in an episode is high $\left(\mathrm{F}_{\mathrm{H}}\right)$, a short-term duration (duration $=t_{1}$ ) should be detected.

- If the magnitude of bite force is medium $\left(\mathrm{F}_{\mathrm{M}}\right)$, the system can detect whether the bruxist event duration is a bit larger than the previous one (duration $=t_{2}$ ).

- If the magnitude of bite force is low $\left(\mathrm{F}_{\mathrm{L}}\right)$, the detection is just made if the episode duration time is sufficiently greater than the previous ones (duration $=t_{3}$ ).

The criteria for determining the high, medium or low magnitudes values and the corresponding episodes time are given by a bruxism specialist dentist based on clinic history of the patient.
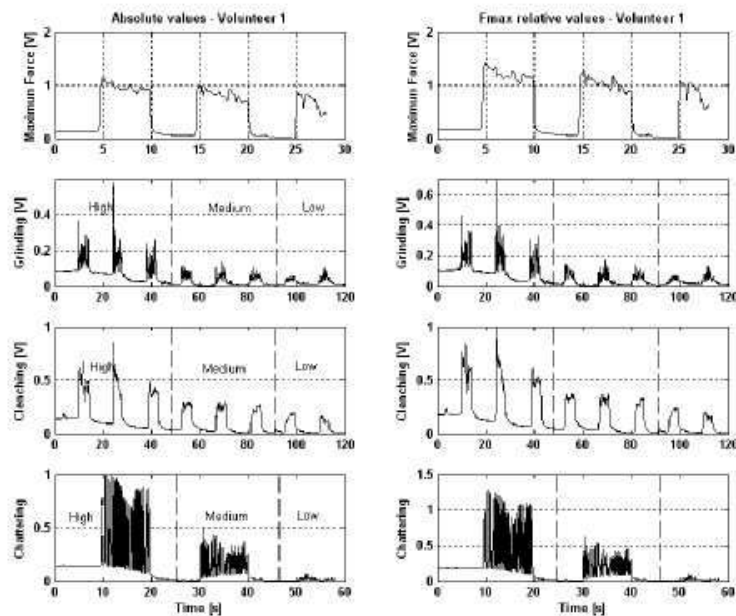

Fig. 5. "In vivo" validation of the system for bruxism detection. 


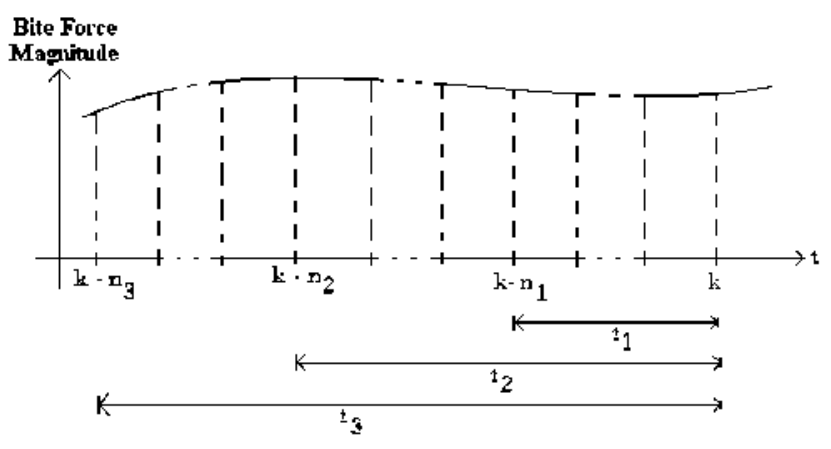

Fig. 6. Deseription of the detection algorithm under basis conditions.

To implement this algorithm we used a summation of samples for different periods of time (shor, medium and long) verifying if this sum reaches or exceeds a certain threshold defined by the values of force previously considered (high, medium or low). According to this and based on Figure 6:

- Activate alarm for event with high magnitude and shortterm duration if satisfies (1).

Where:

$$
\sum_{t\left(k-n_{1}\right)}^{t(k)} f(k)>n_{1} F_{H}
$$

$f(k)$ : magnitude of the bite force sampled signal.

$n_{1}$ : number of samples corresponding to $t_{1}$ ( $t_{1}$ defined by the dentist)

$\mathrm{F}_{\mathrm{H}}$ : Value of high-strength threshold $\left(\mathrm{F}_{\mathrm{H}}\right.$ defined by the dentist)

- Activate alarm for event with medium magnitude and medinm-term dnration if satisfies (2).

Where:

$$
\sum_{t\left(k-n_{2}\right)}^{t(k)} f(k)>n_{2} F_{M}
$$

$\mathrm{f}(\mathrm{k})$ : magnitnde of the bite force sampled signal.

$\mathrm{n}_{2}$ : number of samples corresponding to $\mathrm{t}_{2}\left(\mathrm{t}_{2}\right.$ defined by the dentist)

$F_{M}$ : Value of medium-slrength threshold $\left(F_{M}\right.$ defined by the dentist)

- Activate alann for event with low magnitude and large-tenn duration if satisfies (3).

Where:

$$
\sum_{t\left(k-n_{3}\right)}^{t(k)} f(k)>n_{3} F_{L}
$$

$f(k)$ : magnitude of the bite force sampled signal.

$\mathrm{n}_{3}$ : number of samples corresponding to $t_{3}\left(t_{3}\right.$ defined by the dentist)

$\mathrm{F}_{\mathrm{L}}$ : Value of low-strength threshold $\left(\mathrm{F}_{\mathrm{L}}\right.$ defined by the dentist)
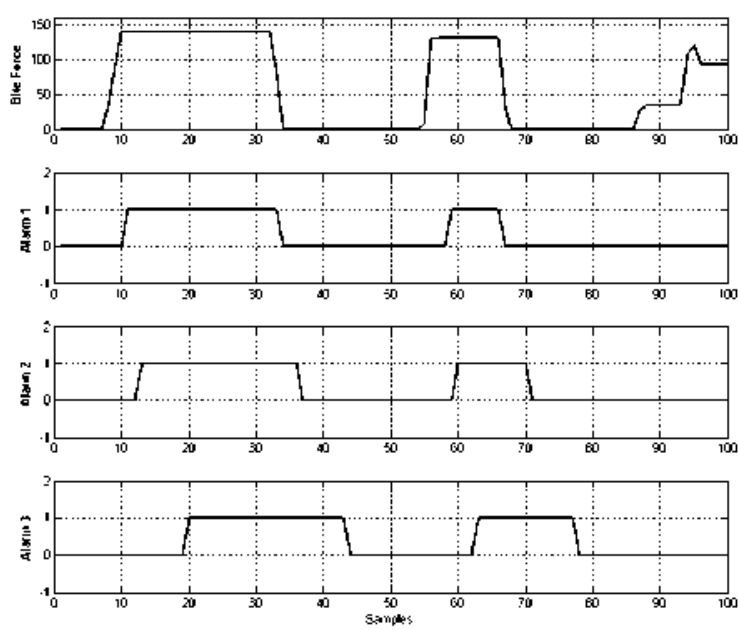

Fig. 7. Applied bite force and alam activation according to the magnitude and lenglh of bruxist episodes.

The software developed from this algorithm runs within the Bluetooth module. For testing, bite forces were applied on an instrumented splint and data were collected on the mobile phone through a Bluetooth connection. The first plot of Fig. 7 represents the magnitude of the applied force, while the other plots show the time in which the alarms were activated according to the algorithm mles, which can be associated in a mobile phone to three different types of stimuli, i.e. vibration, light or sonnd. It may be noted that each of the alams is triggered (from 0 to 1 ) according to the time that force has been applied on sensor.

Once the proper behaviour of the detection algorithm was validated, several trials were carried out, including one test in which a $60 \%$ threshold of the hypothetical maximum bite force was introdnced using the mobile phone.

Using the pnenmatic bite simnlator (shown in Fig. 8) episodes including two "bites" were simulated, at first supplying 2.0 bar to the pneunatic muscles during 2 seconds for each bite. Snbseqnently supply pressure and bite length were increased. Trials include combinations of $2.0,2.5,3.0$ and 4.0 bar, together with $2,4,6,8$, and 10 seconds of application time. Such applied pressnres have been selected according to simnlator's geometry, so as to produce forces on the PVDF sensors similar to those typical of bruxist episodes (around 300 $\mathrm{N}$ to $(200 \mathrm{~N})$. 


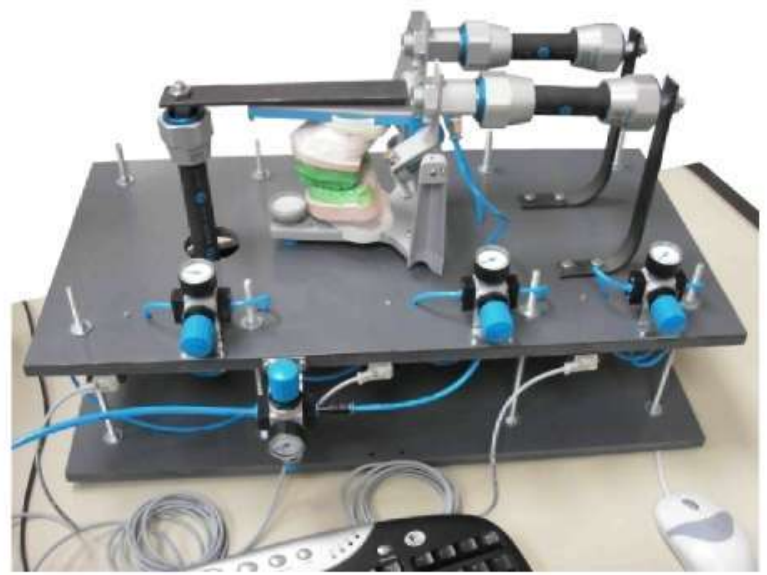

Fig. 8. Pneumatic workbench for simulating bruxist episodes.

Table 1 shows the register of alarm activation during the trials, according to magnitude and length of the bite force applied ("X" indicates activation during an specific episode and " 0 " indicates no activation).

Table 1. Activation of different alarm types, according to magnitude and length of the bite force applied.

\begin{tabular}{||c|c|c|c|c|c|c|c|c|c|c|c||}
\hline & \multicolumn{10}{|c|}{ and length of the bite force applied. } \\
\hline 550.0 & 0 & 0 & 0 & 0 & 0 & 0 & 0 & 0 & 0 & 0 & \\
\hline 687.5 & 0 & 0 & 0 & $\mathrm{X}$ & $\mathrm{X}$ & $\mathrm{X}$ & $\mathrm{X}$ & $\mathrm{X}$ & $\mathrm{X}$ & $\mathrm{X}$ & \\
\hline 825.0 & $\mathrm{X}$ & 0 & $\mathrm{X}$ & $\mathrm{X}$ & $\mathrm{X}$ & $\mathrm{X}$ & $\mathrm{X}$ & $\mathrm{X}$ & $\mathrm{X}$ & $\mathrm{X}$ & \\
\hline 1100.0 & 0 & $\mathrm{X}$ & $\mathrm{X}$ & $\mathrm{X}$ & $\mathrm{X}$ & 0 & $\mathrm{X}$ & $\mathrm{X}$ & 0 & $\mathrm{X}$ & \\
\hline $\begin{array}{c}\text { Applied Force } \\
{[\mathrm{N}]}\end{array}$ & & & & & & & & & & & \\
\hline
\end{tabular}

This table shows that the system does not generate alarms when the applied force is below than the threshold (regardless the time of applied force). Besides, the alarm is generated whether the force exceeds the corresponding threshold Furthermore, the delay time detection is inversely proportional to the magnitude of the applied force.

\section{MAIN CONCLUSIONS}

The complete development of a splint to assess intrabucal pressure and detect bruxism and other occlusive pathologies has been presented.

What should be highlighted is the possibility to obtain a device that will enable intrabucal pressure to be quantitatively assessed and bruxism behaviour to be diagnosed at an early stage, so that corrective actions can be programmed before the appearance of irreversible dental wear.

Additionally, the presented telecommunication capabilities due to inclusion of Bluetooth modules as part of the system's electronics, introduces important advances in the field of remote control and telemedicine tasks, related to the described pathology.

We believe the results can be used for the development of new medical devices for diagnosis aid and treatment of several pathologies, which would benefit from pressure sensing together with the possibilities provided by remote communication.

\section{REFERENCES}

[1] P. Dechow, et al., 2003. "A method of bite force measurement in primates". Journal of Biomechanics, Vol. 16(10), 797-802.

[2] V. Bousdras, et al., 2006. "A novel approach to bite force measurements in a porcine model in vivo". International Journal of Oral and Maxillofacial Surgery, Vol. 35(7), 663-667.

[3] D.L. Lindner, et al., 1995. "Measurement of bite force in dogs: a pilot study". Journal of Veterinary Dentistry, Vol. 12(2), 49-52.

[4] C.P. Fernandes, et al., 2003. "A novel sensor for bite force determinations". Dental Materials, Vol. 19(2):118-126.

[5] M. Rismanchian, E. Mostajeran, 2007. "Evaluation of Maximum Bite Force and Satisfaction in Patients with Conventional Full Denture and Over Denture Supported by Mandibular Dental Implant". Journal of Isfahan Dental School, Vol. 2(4):1358.

[6] D. Tortopidis, et al., 1998. "The variability of bite force measurement between sessions, in different positions within the dental arch". Journal of Oral Rehabilitation, Vol. 25(9), 681-686.

[7] G. Lavigne et al., 1995. "Bruxism: Epidemiology, diagnosis, pathophysiology and pharmacology. Advances in pain research and treatment, Vol. $21,387-404$.

[8] G. Lavigne et al., 2003, "Neurobiological mechanisms involved in sleep bruxism. Critical Reviews in Oral Biology \& Medicine, Vol. 14(1), 30-46.

[9] G. Lavigne et al., 1996. Sleep bruxism: validity of clinical research diagnostic criteria in a controlled polysomnographic study. Journal of Dental Research, Vol. 75(1), 546-552.

[10] M. Melis, Y.S. Abou-Atme, 2003. "Prevalence of bruxism awareness in a Sardinian population". Journal of Craniomandibular Practice, Vol. 21(2), 144-151.

[11] M. Melis, Y.S. Abou-Atme, K.H. Zawawi, 2004. "Bruxism prevalence in a selective Lebanese population". Journal of the Lebanese Dental Associoation, 41(2), 31-35.

[12] K. Nishigawa, et al., 2001. "Quantitative study of bite force during sleep associated bruxism. Journal of Oral Rehabilitation, Vol. 28(5), 485-491.

[13]D. Cosme, et al., 2005. "Bruxism and voluntary maximal bite force in young dentate adults". The International Journal of Prosthodontics, Vol. $18(4), 328-332$.

[14] K. Baba, 2003. "Bruxism force detection by a piezoelectric film-based recording device in sleeping humans". Journal of Orofacial Pain, Vol. $17(1), 58-64$.

[15] P. Lafont Morgado, et al., "System for the diagnosis and detection of bruxism and other occlusal pathologies". PCT/ES2008/000498. PCT Patent Document. July, 15, 2008.

[16] P. Lafont Morgado, et al., 2008. "Sensores piezoeléctricos poliméricos para detección de fuerzas y duraciones de mordidas: Aplicación al tratamiento del bruxismo". Anales de Ingenieria Mecánica, Año 16, Vol. 1, 67-76, 2008.

[17] P. Lafont Morgado, A. Diaz Lantada, et al., 2008. "Instrumented splint for the diagnosis of bruxism". Biodevices 2008 - International Conference on Biomedical Electronics and Devices. IEEE Engineering in Medicine and Biology Society. January, 28-31, Madeira. 